\title{
Efficacy and safety of advanced renal cell carcinoma patients treated with sorafenib: roles of cytokine pretreatment
}

\author{
Hisanori Suzuki $^{1), 2)}$, Toshiro Suzuki ${ }^{1), 2)}$, Osamu Ishizuka ${ }^{1), 2)}$, \\ Osamu Nishizawa ${ }^{1), 2)}$, Manabu Ueno ${ }^{2)}$
}

1) Department of Urology, Shinshu University School of Medicine

3-1-1 Asahi, Matsumoto 390-8621, Japan

2) Shinshu Renal Cancer Study Group

Corresponding Author:

Osamu Ishizuka, M.D., Ph.D.

Department of Urology, Shinshu University School of Medicine

3-1-1 Asashi, Matsumoto 390-8621, Japan

Tel. +81263 372661, Fax. +81263373082

e-mail: ishizuk@shinshu-u.ac.jp 


\begin{abstract}
Background We conducted a retrospective, questionnaire-based analysis to assess the outcomes of advanced renal cell carcinoma (RCC) in Japanese patients treated with sorafenib in a daily clinical setting.
\end{abstract}

Patients and methods Patients $(\mathrm{n}=110)$ were treated with sorafenib $400 \mathrm{mg}$ twice daily at 12 centers. Overall survival (OS), progression-free survival (PFS), safety, and prognosis factors associated with PFS were assessed.

Results The median OS was not yet reached within the study period while the median PFS was 11.0 mo [95\% confidence interval (CI), 6.6 to 14.4 mo]. Univariate analysis showed that higher CRP level, lower Na level, and presence of liver metastasis were significant predictors of poorer PFS ( $p<0.05$, respectively). Among these variables, multivariate analysis identified higher CRP levels $(\mathrm{p}=0.004)$ and the presence of liver metastasis $(p<0.001)$ to be significantly associated with poorer PFS .The most common adverse event was skin toxicity (67\%), followed by gastrointestinal symptoms (26\%), hypertension (22\%), fatigue (19\%), hematological toxicity (10\%), and hemorrhage (6\%). The incidence of adverse events was comparable to that of previously reported clinical trials. 
Conclusions Multivariate analysis indicated that CRP and liver metastasis were negatively associated with prognosis. Sorafenib therapy for Japanese patients with advanced RCC was effective and well tolerated.

$198 / 250$ words 


\section{Mini abstract}

A retrospective study to assess the outcomes of advanced renal cell carcinoma in

Japanese patients treated with sorafenib in a daily clinical setting confirmed the efficacy and safety of sorafenib.

$30 / 30$ words

Keywords: advanced renal cell carcinoma, sorafenib, overall survival, progression free survival, prognosis factors 


\section{Introduction}

Tyrosine kinase inhibitors (TKIs) are a standard therapeutic option for metastatic

renal cell cancer (RCC). Sorafenib is an oral tablet that inhibits receptor tyrosine kinases, such as VEGFR2, VEGFR-3, Raf, PDGFR $\beta$, Flt3, and c-Kit, that are associated with tumor cell proliferation and angiogenesis [1,2]. This inhibitor was an effective treatment for unresectable or metastatic RCC in clinical trials conducted in and out of Japan $[3,4]$. Sorafenib became available on the Japanese market in April 2008, and it has been widely used for treatment of advanced RCC.

Recent findings of the phase II trial on efficacy and safety in Japanese patients with advanced RCC showed that long-term use of sorafenib after cytokine therapy is well tolerated and prolongs survival [5]. However, the phase II trial study population included only patients in which cytokine therapy failed; therefore the results cannot be safely generalized to include daily clinical practice. Sorafenib is currently used as a first-line therapy in addition to a second-line therapy after cytokine or other TKI treatments. Thus, confirming the effectiveness of sorafenib in daily clinical practice is essential. To investigate the efficacy and safety of sorafenib for Japanese patients with advanced RCC under daily clinical practice, we retrospectively analyzed the available data of such patients at 12 centers in Nagano and Yamanashi Prefectures, Japan. 


\section{Patients and Methods}

Patients and study design

This multicenter, retrospective analysis of sorafenib was conducted at 12 centers in Nagano and Yamanashi Prefectures, Japan (see Appendix) and was approved by the ethics committee of Shinshu University School of Medicine. A total of 110 Japanese patients with advanced RCC treated by sorafenib were included. Follow-up started on the first administration of sorafenib, and it ended on August 1, 2012 or upon the withdrawal or death of the patient. Administration of sorafenib was based on the approved procedure by the Ministry of Health, Labour and Welfare of Japan. Patients received oral sorafenib (Nexavar ${ }^{\mathrm{R}}$; Bayer HealthCare, Oosaka, Japan) $400 \mathrm{mg}$ twice daily on a continuous basis, and an increase of dosage was not permitted.

Administration of sorafenib was discontinued if the patient had progressive disease, benefit of further sorafenib therapy was improbable, or sorafenib therapy was not tolerated by the patient. For those patients, the other drugs, such as sunitinib, everolimus, temsirolimus were used for the treatment.

Patient clinical data were collected through a questionnaire that included the following information: patient background information, histological information, treatment history of TKIs, and outcome. Patient background information included 
gender, age, date of diagnosis, medical history, history of previous use of cytokine or TKIs, Memorial Sloan-Kettering Cancer Center (MSKCC) risk criteria, Karnofsky performance status (KPS), as well as the results of hematologic tests. Information on the treatment history with TKIs included duration of therapy, dose intensity, and outcome. The questionnaires were sent to all 12 centers on July 31, 2012. Results from the questionnaires that were returned by September 30, 2012, are presented herein.

\section{Outcomes}

The primary endpoint of the present study was overall survival (OS), and the secondary endpoints were progression-free survival (PFS) and safety. For safety, information on adverse events from the following categories were collected: 1) skin toxicity, including hand-foot syndrome; 2) hematological toxicity, including abnormal changes in white blood cell count, platelet count, or hemoglobin level; 3) hypertension; 4) hemorrhage; 5) gastrointestinal symptoms; and 6) fatigue. 
Patient background information was summarized in a descriptive manner. The median OS and PFS were estimated using the Kaplan Meier method and a log-rank test was used to compare survival curves. To evaluate the prognostic factors of survival, univariate and multivariate Cox regression analyses were also performed. P-values of $<0.05$ were considered to indicate statistically significant differences. 


\section{Results}

Patient backgrounds

There were 83 men and 27 women with a median age of 67 years (range, 31 to 84 years, Table 1). Eighty-one patients (73.6\%) had clear cell carcinoma and 100 patients $(91.0 \%)$ were in stage IV. Based on the MSKCC risk classification, the majority of patients were classified as favorable (34.5\%) and intermediate (58.2\%). Only 8 patients $(7.3 \%)$ were classified as having poor risk. Forty-four patients $(40.0 \%)$ were treated with a TKI as a first-line therapy. Among them, 40 patients $(91.0 \%)$ received sorafenib. During the study period, more than half of the patients $(56.4 \%)$ were able to continue sorafenib therapy without dose reduction. The median duration of sorafenib therapy was 470 days (range, 35 to 1631 days). The most common metastasis sites (Table 2) were lung (65.5\%), lymph node (35.5\%), bone (19.1\%), and liver (11.8\%).

The figure 1 shows the difference of nephrectomy, previous cytokine therapy, and the treatment order of sorafenib (Fig. 1).

Overall survival

Among all 110 patients, the median OS was not yet reached within the study period, and the 1-year survival rate was $77.5 \%$ [95\% confidence interval (CI), 67.8 to $84.6 \%$ ] 
(Figure 2a). The median OS was not reached for patients with normal C-reactive protein (CRP) levels or for patients with no liver metastasis. For patients with higher CRP levels, the median OS was 18.0 mo (95\% CI, 11.4 to not applicable:NA, $\mathrm{p}=0.034)$ and 18.6 mo $(95 \%$ CI, 3.3 to NA, $p=0.005)$ for patients with liver metastasis. Moreover, the median OS for patients with favorable risk, based on the MSKCC risk classification, was not reached. It was significantly higher compared to the OS of $22.5 \mathrm{mo}(95 \% \mathrm{CI}$, 14.3 to NA) for patients with intermediate and poor risks $(p=0.025)$ based on the MSKCC risk classification (Figure 2b). It was also significantly higher OS (95\%CI, 39.8 to NA) for patients with cytokine therapy before sorafenib $(n=62)$ than without cytokine therapy (n=37) (Fig.1, Fig 2c, p=0.002). And it was also significantly higher OS (95\%CI, NA to NA) for nephrectomy patients with cytokine therapy before sorafenib $(n=55)$ than without cytokine therapy $(n=24)$ (Fig.1, Fig 2d, p=0.034). However it was no significant difference of OS (95\%CI, NA to NA) between patients clear cell carcinoma $(n=81)$ and non-clear cell carcinoma $(n=10)$ before sorafenib (Fig.1, Fig $2 \mathrm{e}, \mathrm{p}=0.487)$. 
Among all 110 patients, the median PFS was 11.0 mo (95\% CI, 6.6 to 14.4; Figure 3a).

The median PFS was 11.0 mo (95\% CI, 6.6 to 16.8$)$ for patients who had KPS $\geq 80 \%$ and 3.5 mo $(95 \%$ CI, 1.5 to NA) for patients who had KPS $<80 \%(p=0.022)$. For patients with liver metastasis the media PFS was 3.1 mo (95\% CI, 1.6 to 5.5) and 12.3 mo (95\% CI, 7.5 to 20.1 ) without liver metastasis ( $<<0.001$ ). For patients with lymph node metastasis (39 out of 110, table 2), the median PFS was 5.9 mo (95\% CI, 3.9 to 17.1) and $13.8 \mathrm{mo}(95 \% \mathrm{CI}, 7.5$ to 29.3$)$ without lymph node metastasis $(\mathrm{p}=0.037)$. For patients with above normal CRP levels, the mean PFS was 6.1 mo (95\% CI, 4.4 to 12.1 , $\mathrm{p}=0.012$ and $14.3 \mathrm{mo}(95 \% \mathrm{CI}, 8.1$ to 31.2$)$ for patients with normal CRP levels. For patients with normal $\mathrm{Na}^{+}$levels, the mean PFS was 11.0 mo (95\% CI, 6.9 to 17.1$)$ and 4.8 mo $\left(95 \% \mathrm{CI}, 0.1\right.$ to NA) for patients with low $\mathrm{Na}^{+}$levels $(\mathrm{p}=0.042)$. The median PFS (95\% CI, 5.9 to 24.4) for patients with favorable risk factors was $14.3 \mathrm{mo}$, which was not significantly longer than the $7.5 \mathrm{mo}(95 \% \mathrm{CI}, 5.6$ to $13.8, \mathrm{p}=0.253)$ for patients with intermediate and poor risks (Figure 3b). It was also significantly higher PFS of $17.1 \mathrm{mo}(95 \% \mathrm{CI}, 6.9$ to 29.3$)$ for patients with cytokine therapy before sorafenib $(\mathrm{n}=62)$ than without cytokine therapy $(n=37)$ (Fig.1, Fig 3c, $p=0.017)$. However it was no significant difference of the PFS between nephrectomy patients with cytokine therapy $(n=55)$ and without cytokine therapy $(n=24)$ before sorafenib (Fig.1, Fig 3d, $p=0.091)$. 
And it was significantly higher PFS of $12.1 \mathrm{mo}(95 \% \mathrm{CI}, 6.9$ to 24.4$)$ for patients clear cell carcinoma $(n=81)$ than non-clear cell carcinoma $(n=10)$ (Fig.1, Fig 3e, $p<0.001)$.

\section{Univariate and multivariate analysis}

Univariate analysis showed that higher CRP level, lower Na level, and presence of liver metastasis were significant predictors of poorer PFS ( $\mathrm{p}<0.05$, respectively). Among these variables, multivariate analysis identified higher CRP levels $(p=0.004)$ and the presence of liver metastasis $(\mathrm{p}<0.001)$ to be significantly associated with poorer PFS (Table 3).

Adverse events

We analyzed the adverse events in 110 cases. The most common adverse event was skin toxicity (67\%). Other adverse events were gastrointestinal symptoms (26\%), hypertension (22\%), fatigue (19\%), hematological toxicity (10\%), and hemorrhage (6\%).

Grade 3 side events were skin toxicity 5 cases, gastrointestinal symptom (diarrhea, pancreatitis) 4 cases, severe general fatigue 5 cases, thrombocytopenia 1 case, heart 
failure 1 case, interstitial pneumonia 1 case, lung bleeding 1 case. All events were recovered by stopping sorafenib. 


\section{Discussion}

In the present study, the clinical data of 110 Japanese patients with advanced RCC treated with sorafenib in a daily clinical setting were retrospectively analyzed. Sorafenib became available on the market in Japan later than in western countries, and thus there is less evidence for the efficacy and safety of sorafenib therapy for advanced RCC in Japanese patients. Therefore analyzing data from daily clinical settings is important. In addition, finding the prognostic factors that are associated with sorafenib therapy is essential.

Currently, a post-marketing surveillance of sorafenib for advanced RCC in Japan is completed, and final data is available [6]. According to the data of 3,171 patients, the median PFS is 7.3 mo (95\% CI, 6.8 to $8.2 \mathrm{mo})$, and the one-year survival rate is $75.4 \%$ (95\% CI, 73.5 to $77.1 \%$ ). The final results of the European Advanced Renal Cell Carcinoma Sorafenib (EU-ARCCS) expanded-access study showed that the median PFS was 6.6 mo [7], and the final results of the North American Advanced Renal Cell Carcinoma Sorafenib (NA-ARCCS) expanded-access study showed that the median PFS was 9.0 mo [8]. Although there were differences in patients' backgrounds and study protocols between the present study and the other studies described above, the efficacy of sorafenib in the present study was not much different compared to them. 
The efficacy data of pre-marketing phase II/III studies showed that the median OS and PFS were 25.3 and 7.9 mo respectively in the Japanese phase II study [5], and 17.8 and 5.5 mo in the Treatment Approaches in Renal Cancer Global Evaluation Trial (TARGET) study [3,9]. Compared to those results, the present data indicated better survival. Before sorafenib became available in Japan, the median OS of Japanese patients with metastatic RCC treated with cytokine therapy was 24.9 mo [10]. Although we cannot directly compare the results of different studies, sorafenib therapy could be comparable or more effective for patients with advanced RCC compared to cytokine therapy. And the important points of this study, although cytokine pretreated group may contain more favorable risk patients than the counterpart, cytokine pre-treatment may contibuted the good OS and PFS.

In our multivariate analysis, two factors, higher CRP levels and presence of liver metastasis, were significantly associated with short PFS. Systemic inflammation is associated with the cancer progression, and factors that are associated with inflammation have been considered as potential biomarkers $[11,12]$. CRP is an acute-phase reactant protein exclusively synthesized by hepatocytes. The serum concentration of CRP rises as much as 1000-fold in immediate response to cytokines or chemical mediators released in various pathological conditions, including acute 
inflammation, infection, tissue or cell necrosis, and some malignancies including RCC.

Previously reported studies indicated that higher CRP levels are significantly associated with poor OS in patients with advanced RCC [11-16]. Thus, CRP can be used as an indicator of patient outcome, and monitoring CRP levels might help to predict the disease course.

RCC metastasis to liver is widely known to be one of the major factors of poor outcome. Although sorafenib therapy is likely to be more effective than conventional cytokine therapy, liver metastasis still affects prognosis. On the other hand, the efficacy of sorafenib in patients who had lung, lymph node, and bone metastasis was similar to those who did not. These results suggest that sorafenib may be beneficial for the overall outcome for patients with RCC with metastasis other than to liver.

The incidence rate of adverse events reported in the present study was expected and comparable to previously reported clinical studies [5,7-9]. However, information on grading of adverse events in the present study was absent.

Here we reported essential clinical data in daily use of sorafenib for the treatment of advanced RCC; however, there were some limitations. The clinical data was collected by questionnaire and analyzed retrospectively. Due to the small number of samples and study design, the results are not directly comparable to those from large-scale 
randomized controlled studies. The safety data was limited to the following six categories: skin toxicity including hand-foot syndrome, hematological toxicity, hypertension, hemorrhage, gastrointestinal symptoms, and fatigue, which are known to be common adverse events of sorafenib therapy. We were unable to collect other adverse events which might be clinically important but with low incidence.

In summary, the present retrospective study showed additional clinical information on sorafenib therapy for the treatment of advanced RCC in daily clinical settings.

Survival of patients in the present study was better than that previously reported while safety of sorafenib therapy was comparable. In addition, multivariate analysis indicated that higher CRP levels and presence of liver metastasis were significantly associated with short PFS. 


\section{Conflict of interest}

The authors declare that there are no conflicts of interest. 


\section{Appendix}

\begin{tabular}{|c|c|}
\hline Affiliation & Name \\
\hline \multirow{4}{*}{ Shinshu University Hospital } & Osamu Nishizawa \\
\hline & Osamu Ishizuka \\
\hline & Hisanori Suzuki \\
\hline & Toshiro Suzuki \\
\hline \multirow{2}{*}{ Nagano Municipal Hospital } & Azusa Yamagishi \\
\hline & Masahiro Gotoh \\
\hline \multirow{2}{*}{ Saku Central Hospital } & Sayo Suda \\
\hline & Manabu Tsukada \\
\hline \multirow[t]{2}{*}{ Shinonoi General Hospital } & Manabu Ueno \\
\hline & Kyoko Hosaka \\
\hline \multirow[t]{2}{*}{ Yamanashi Prefectural Central Hospital } & Shiro Hiragata \\
\hline & Ataru Suzuki \\
\hline Ina Central Hospital & Tsuyoshi Nakayama \\
\hline Iida Municipal Hospital & Yuji Mimura \\
\hline Shinshu Ueda Medical Center & Tetsuichi Saito \\
\hline Japanese Red Cross Society Suwa Hospital & Takahisa Domen \\
\hline Matsumoto Medical Center & Hiroo Inoue \\
\hline Hokushin General Hospital & Yuji Shimojima \\
\hline Japanese Red Cross Society Azumino Hospital & Akino Tsunekage \\
\hline
\end{tabular}




\section{References}

1. Ratin MJ, Eisen T, Stadler WM, et al. (2006) Phase II placebo-controlled randomized discontinuation trial of sorafenib in patients with metastatic renal cell carcinoma. J Clin Oncol 24: 2505-2512

2. Rini BI (2009) Vascular endothelial growth factor-targeted therapy in metastatic renal cell carcinoma. Cancer 115: 2306-2312

3. Escudier B, Eisen T, Stadler WM, et al. (2007) Sorafenib in advanced clear-cell renal cell carcinoma. N Engl J Med 356:125-134

4. Akaza H, Tsukamoto T, Murai M, et al. (2007) Phase II study to investigate the efficacy, safety, and pharmacokinetics of sorafenib in Japanese patients with advanced renal cell carcinoma. Jpn J Clin Oncol 37:755-762

5. Naito S, Tsukamoto T, Murai M, et al. (2011) Overall survival and good tolerability of long-term use of sorafenib after cytokine treatment: final results of a phase II trial of sorafenib in Japanese patients with metastatic renal cell carcinoma. BJU Int. 108:1813-1819

6. Bayer Yakuhin, Ltd. Final analysis of the post-marketing surveillance of Nexavar ${ }^{\circledR}$ tablet $200 \mathrm{mg}$. October, 2012

7. Beck J, Procopio G, Bajetta E, et al. (2011) Final results of the European Advanced Renal Cell Carcinoma Sorafenib (EU-ARCCS) expanded-access study: a large open-label study in diverse community settings. Ann Oncol 22:1812-1823

8. Stadler WM, Figlin RA, McDermott DF, et al. (2010) Safety and efficacy results of the advanced renal cell carcinoma sorafenib expanded access program in North America. Cancer 116:1272-1280

9. Escudier B, Eisen T, Stadler WM, et al. (2009) Sorafenib for treatment of renal cell carcinoma: Final efficacy and safety results of the phase III treatment approaches in renal cancer global evaluation trial. J Clin Oncol 27:3312-3318

10. Naito S, Yamamoto N, Takayama T, et al. (2010) Prognosis of Japanese metastatic renal cell carcinoma patients in the cytokine era: a cooperative group report of 1463 patients. Eur Urol 57:317-325

11. Yasuda Y, Saito K, Yuasa T, et al. (2012) Prognostic impact of pretreatment $\mathrm{C}$-reactive protein for patients with metastatic renal cell carcinoma treated with tyrosine kinase inhibitors. Int J Clin Oncol [Epub ahead of print]

12. Steffens S, Köhler A, Rudolph R, et al. (2012) Validation of CRP as prognostic marker for renal cell carcinoma in a large series of patients. BMC Cancer 12:399 
13. Saito K, Kihara K (2010) Role of C-reactive protein as a biomarker for renal cell carcinoma. Expert Rev Anticancer Ther 10:1979-1989

14. Shinohara N, Abe T, Mochizuki T, et al. (2011) Is Memorial Sloan-Kettering cancer center risk classification appropriate for Japanese patients with metastatic renal cell carcinoma in the cytokine era? Urol Oncol Sep 26 [Epub ahead of print]

15. Miyake H, Kurahashi T, Takenaka A, et al. (2009) Clinical outcome of combined immunotherapy with interferon-alpha and low-dose interleukine-2 for Japanese patients with metastatic renal cell carcinoma. Urol Oncol 27: 598-603

16. Ito H, Shioi K, Murakami $\mathrm{T}$ et al. (2012) C-reactive protein in patients with advanced metastatic renal cell carcinoma: Usefulness in identifying patients most likely to benefit from initial nephrectomy. BMC cancer 12:337 


\section{Figure Legends}

\section{Fig. 1}

The difference of nephrectomy, previous cytokine therapy, and the treatment order of sorafenib.

Fig. 2

Probability estimates of overall survival (OS). (a) The one-year survival rate for patients $(n=110)$ treated with sorafenib was $77.5 \%$, and the median OS was not yet reached in the study population. (b) For patients classified as having favorable (F) risk for survival according to the Memorial Sloan-Kettering Cancer Center (MSKCC) classification, the one-year survival rate was $88.5 \%$, and the median OS was not yet reached. For patients classified as having intermediate or poor (IP) risk for survival, the one-year survival rate was $71.2 \%$, and the median OS was 22.5 mo. (c) For patients classified as with cytokine therapy before sorafenib regardless of nephrectomy. The one-year survival rate was $84.0 \%$ and the median OS was not reached. For patients classified as without cytokine therapy before sorafenib regardless of nephrectomy. The one- year survival rate was $63.9 \%$ and the median OS was 21.0 mo . (d) For patients classified as with cytokine therapy before sorafenib with nephrectomy. The one-year survival rate was $83.9 \%$ and 
the median OS was not reached. For patients classified as without cytokine therapy before sorafenib with nephrectomy. The one- year survival rate was $72.8 \%$ and the median OS was 30.4 mo (e) For patients with clear cell carcinoma. The one-year survival rate was $82.9 \%$, and the median OS was not reached For patients with non-clear cell carcinoma. The one-year survival rate was $64 \%$, and the median OS was not reached.

\section{Figure 3.}

Probability estimates for progression-free survival (PFS). (a) The median one-year PFS rate for patients $(\mathrm{n}=110)$ treated with sorafenib was $46.3 \%$, and the median PFS was 11.0 mo. (b) For patients classified as having favorable (F) risk for survival according to the Memorial Sloan-Kettering Cancer Center (MSKCC) classification, the one-year PFS rate was $57.8 \%$, and the median PFS was 14.3 mo. For patients classified as having intermediate or poor (IP) risk for survival, the one-year PFS rate was $39.5 \%$, and the median PFS was 7.5 mo. (c) For patients classified as with cytokine therapy before sorafenib regardless of nephrectomy. The one-year survival rate was $60.0 \%$, and the median PFS was 17.1 mo. For patients classified as without cytokine therapy before sorafenib regardless of nephrectomy. The one-year survival rate was $24.9 \%$, and the 
median PFS was7.53 mo. (d) For patients classified as with cytokine therapy before sorafenib with nephrectomy. The one-year survival rate was $57.9 \%$, and the median PFS was 20.1 mo. For patients classified as without cytokine therapy before sorafenib with nephrectomy. The one-year survival rate was $26.2 \%$, and the median PFS was7.77 mo. (e) For patients with clear cell carcinoma. The one-year survival rate was $49.1 \%$, and the median PFS was 12.1mo. For patients with non-clear cell carcinoma. The one-year survival rate was $15.2 \%$, and the median PFS was 2.93 mo. 